\title{
Data Sharing of Mechanical Design Formulas Using Semantic Web Technology*
}

\author{
Jun ZHOU $^{* *}$ and Keiichi WATANUKI ${ }^{* *}$ \\ ${ }^{* *}$ Graduate School of Science and Engineering, Saitama University \\ 255 Shimo-okubo, Sakura-ku, Saitama-shi, Saitama 338-8570, Japan \\ E-mail: \{shuu, watanuki\}@mech.saitama-u.ac.jp
}

\begin{abstract}
Speed and efficiency are necessary in the field of mechanical design. $\mathrm{CAD} / \mathrm{CAM} / \mathrm{CAE}$ technologies have advanced and attention has also been paid to increasing the efficiency of data sharing and agent processes in the web environment. In this paper, Semantic Web technology is used to enable the sharing of metadata. The metadata consists of design documents and design formulas, with additional semantic information inserted. Mathematical information is expressed by adding metadata into conventional mechanical design formulas using a Resource Description Framework (RDF). The design formulas are later written in MathML (Mathematical Markup Language) for the sake of data sharing. In this way, data sharing and advanced searching is made easy, because the relevant information is made machine readable in the web environment. The calculation of design formulas is made possible using a mathematical processing system, thus increasing the efficiency of mechanical design.
\end{abstract}

Key words: Semantic Web, RDF, MathML, RDFS, Mechanical Design Formula, Metadata, Data Sharing

\section{Introduction}

At present, the Internet has become one of the main tools for information exchange. Searching for appropriate information is therefore an important subject for Internet developers. Due to the rapid development of computer and Internet technology, the amount of information is growing rapidly, thus exacerbating the difficulty in obtaining valuable information from the web.

Accordingly, Tim Berners-Lee ${ }^{(1)}{ }^{(2)}$ has suggested that the existing web evolve a Semantic Web. Semantic Web technology defines the entire web using metadata, and a software agent takes the role of humans in processing metadata on the web. In this way, advanced searching and sharing of information on the Internet is made possible. Researches for the purpose of knowledge sharing and reusing have being actively wrestled with in recent years. For example, researches related to knowledge managements such as Formal languages for Knowledge Engineering ${ }^{(3)}$ approach, Problem-Solving Methods ${ }^{(4)}$ are done. Still more, in order to achieve a more advanced knowledge sharing by taking advantage of ontology technology ${ }^{(5)}, \mathrm{HPKB}^{(6)}$ and IBROW3 ${ }^{(7)}$ are promoted as projects for a purpose of operating problem solving procedure cooperatively in distributed environments. These researches have contributed to knowledge sharing.

Currently, the majority of formulas included in Web pages are converted into Compuserve Graphics Interchange Format (GIF) images and added on top of the text ${ }^{(8)}$. The images have a high data volume, and corrections are also a large burden. Moreover, information regarding the meaning of formulas might be lost by retrieving the image data 
when the formulas are retrieved. Automatic processing corresponding to the content of formulas such as automatic extraction of metadata is therefore difficult.

However, although it has become possible to describe multimedia resources such as existing HTML documents and images using metadata technology ${ }^{(9)}$ which has partially enabled the expression of knowledge contained in the resources, methods for expressing and sharing resources containing design documents and mathematical formulas have yet to be investigated ${ }^{(10)}$. Existing Semantic Web research has generally placed an emphasis on the importance of classifying and correlating knowledge according to a hierarchical structure. However, the technical issues of selecting which structures to use and how to describe design documents have not yet been discussed in detail.

In this paper, semantic information is attached as metadata to both design documents and design formulas for the purpose of sharing the information they contain. Firstly, the design formulas are written in MathML (Mathematical Markup Language) ${ }^{(11)}$ but later Resource Description Framework (RDF) ${ }^{(12)}$ and RDF Schema (RDFS) ${ }^{(13)}$ documents are attached to enable the sharing of design formulas. As a result of making this information machine readable, not only data sharing, but also high speed search and retrieval of information are made possible. Web pages that contain design formulas are linked to a mathematical processing software called Mathematica ${ }^{(14)}$, whereby their calculation process is simplified, thus increasing the efficiency of mechanical design.

\section{Semantic Web}

\subsection{Semantic Web technology}

The World Wide Web is currently based primarily on documents written in HTML. There is also no way to express which pieces of information are bound together in describing a discrete item, distinct from other items perhaps listed on the same web page.

The Semantic Web is a project that intends to create a universal medium for information exchange by putting documents with meaning (semantics) that may be processed by a computer on the World Wide Web. The Semantic Web uses descriptive technologies, such as RDF and RDFS. These technologies are combined in order to provide descriptions that supplement or replace the content of web documents. Thus, content may be manifested as descriptive data stored in web-accessible databases, or as markup information within documents. The machine-readable descriptions enable content managers to add meaning to the content, thereby facilitating automated information gathering and research by computers.

\subsection{Metadata description language RDF and RDFS}

Metadata are data that describe other data. Generally, a set of metadata describes a single set of data, called a resource. RDF and RDF Schema, which are located at the lowest class layer of the Semantic Web, constitute a language used to describe metadata. Metadata is data that describes the meaning of the HTML document. RDF expresses the metadata that enables machine processing. However, the description of RDF may enable the machine to understand only the basic meaning of a given HTML document. To enable the machine to understand more complicated meanings in HTML documents, content must first be defined using hierarchically structured metadata. In short, it is the metadata technology that supports the Semantic Web.

In the Semantic Web, RDF is used as the foundation for metadata processing. By attaching RDF metadata to HTML documents, a person may read and understand the HTML document while the machine may understand the RDF metadata and may perform appropriate tasks. RDF is a language for expressing information regarding web resources. Its metadata description method is written in the extensible markup language (XML) ${ }^{(15)}$. 
The RDF data model consists of three object types, namely resources, properties and statements. 'Resources' refers to all the objects being described by RDF expressions. A resource may be an entire web page or a part of a web page. A 'property' is a specific aspect, characteristic, attribute, or relation used to describe a resource. An RDF 'statement' is a specific resource together with a named property and the value of that property for the resource. RDF documents describe the attributes of resources and the relationships among the resources. RDF Schema is the basic mechanism used to define the attributes and categories of common resources. RDF Schema is therefore used in order to express design knowledge and to define technical terms and relationships in detail. With the RDFS document, exact data or information can be identified from the Internet through automatic recognition.

\subsection{RDF data model}

Figure 1 shows an RDF data model. The resource is specified inside the ellipse, an attribute is associated with the arrow, and a description is specified inside the rectangle. In this data model, the expression of knowledge is unified in the following sense: the title of the http://www.xxx.ac.jp/design/shaft/shaft_strength.html web page is "The formulas for shaft strength".

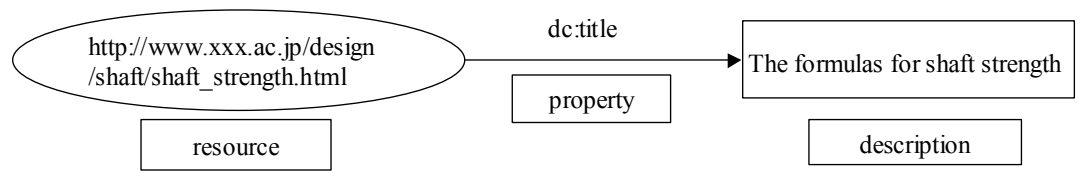

Figure 1 RDF data model

List 1 RDF document

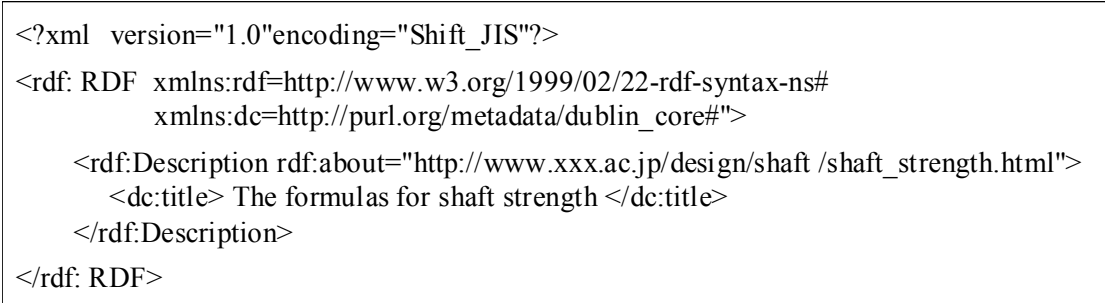

List 1 shows an RDF document for Figure 1. An XML declaration appears first, and the RDF namespace is declared afterwards. The phrase $d c$ reveals that title is metadata defined by Dublin Core ${ }^{(16)}$. The Dublin Core metadata element set is a standard for the cross-domain information resource description. Line 5 in the RDF document begins a description element that may be thought of as simply a place to hold the identification of the resource being described. When the about attribute is specified with description, the statements in the description refer to the resource whose identifier is determined from the about identifier.

\subsection{Design of a strong shaft and associated metadata}

Semantic Web technology defines the entire web using metadata and a software agent replaces the role of humans in processing metadata. As an example, when we would like to search for "The relations between bending stress of a shaft and its diameter" in current search engines, we might use "bending stresses of shaft" and "diameter" as keywords. The results returned may include "diameter" in a context which is related to "bending stresses of shaft", but also "diameter" in a context which has no relation at all with "bending stresses of shaft". By having a set of metadata that are shared by both "bending stresses of shaft" and 
"diameter" according to a relationship described by an RDF Schema document, more accurate search results can be obtained. Therefore, in this paper, we describe the relationship between the formulas for shaft strength and the associated metadata using an RDF document and RDF Schema.

Figure 2 shows a web page that contains formulas for shaft strength and associated metadata. Here the metadata consists of "bending stress" associated with $\sigma$, "diameter" with $d$, "bending moment" with $M$, "shearing stress" with $\tau$ and so on. Figure 3 shows an example of RDF Schema that depicts relationships among classes. We first define the "formulas for shaft strength" as derived from the parent class rdfs: resource. We further define "bending stress", "diameter" and "bending moment", which are derived from the parent class of the "formulas for shaft strength".

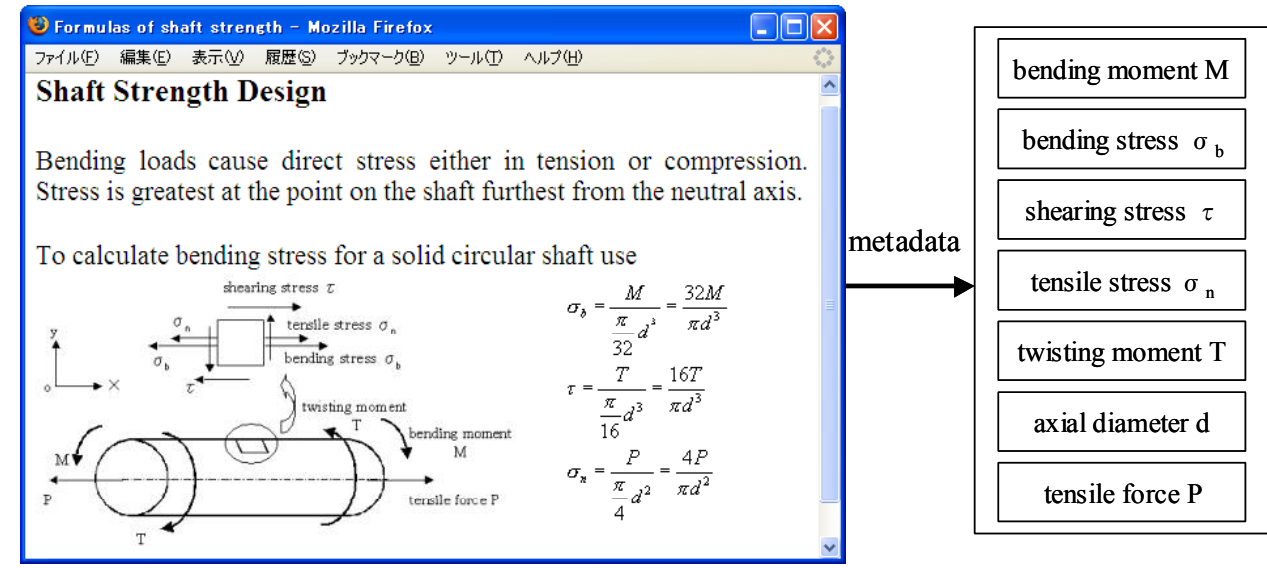

Figure 2 Metadata of shaft strength design

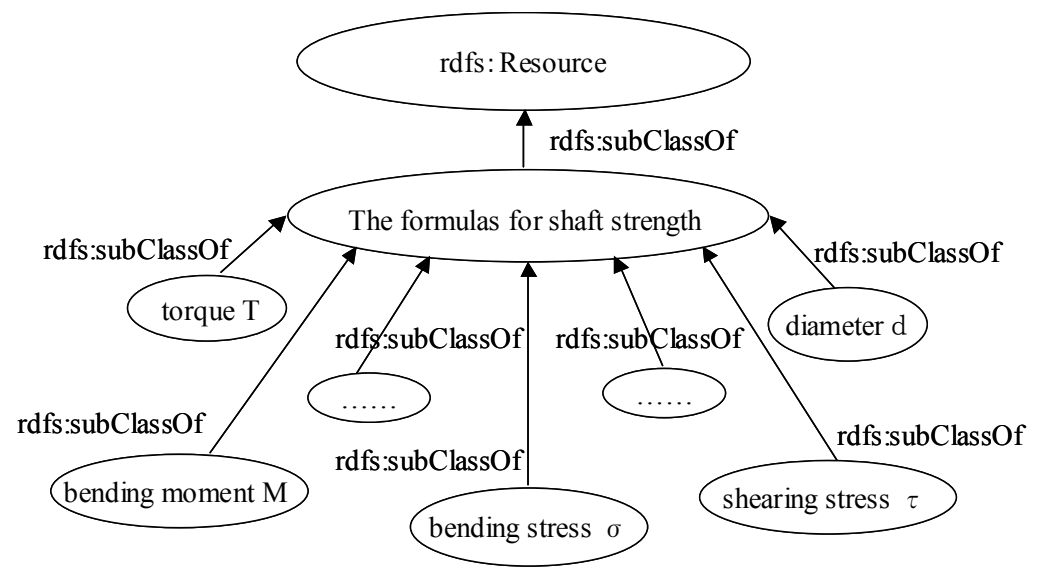

Figure 3 RDF Schema class

List 2 shows part of an RDFS document involving the relationship between the formulas for shaft strength and the associated metadata on the web. Each component of List 2 is explained below.

The first part is the declaration of the rdf and rdfs namespaces using the XML syntax of RDF.

The second part is the description of the "formulas for shaft strength" derived from its parent class rdfs:resource, using a similar RDF Schema document. The rdf:Description element describes the subjects of embedded sentences. In this case "formulas for shaft strength" is considered the subject. The rdf:type element is the identifier of the resource class. The attribute "resource" points to a class that possesses all the characteristics of a 
particular class. For this example, the class "formulas for shaft strength" is a class that possesses all the characteristics of the class http:/www.w3.org/2000/01/rdf-schema\#Class. The rdfs:subClassOf element is used to derive a new class from a parent class. The new class will inherit the characteristics of its parent class. In this example, the class "formulas for shaft strength" has the element rdfs:subClassOf, which makes it a subclass of the class indicated by rdfs:resource.

The third part is also described using an RDFS document, according to which the class "bending stress of shaft $\sigma$ " is derived from its parent class "formulas for shaft strength".

In this example, the rdf:type element has the resource http://www.w3.org/2000/01/rdfschema\#Class that has all the characteristics of the class. Following this is the class "formulas for shaft strength", which has "bending stresses of shaft $\sigma$ " as its subclass as indicated by the element rdfs:subClassOf.

In the web page concerning "formulas for shaft strength", when we define bending stress of shaft $\sigma$, shearing stress $\tau$, diameter $d$ and bending moment $M$, as derivatives from the parent class "formulas for shaft strength", and describe their relationships using RDF Schema document, we enable the machine to recognize the meaning of the web page and facilitate searching and sharing of information.

List 2 RDF Schema document

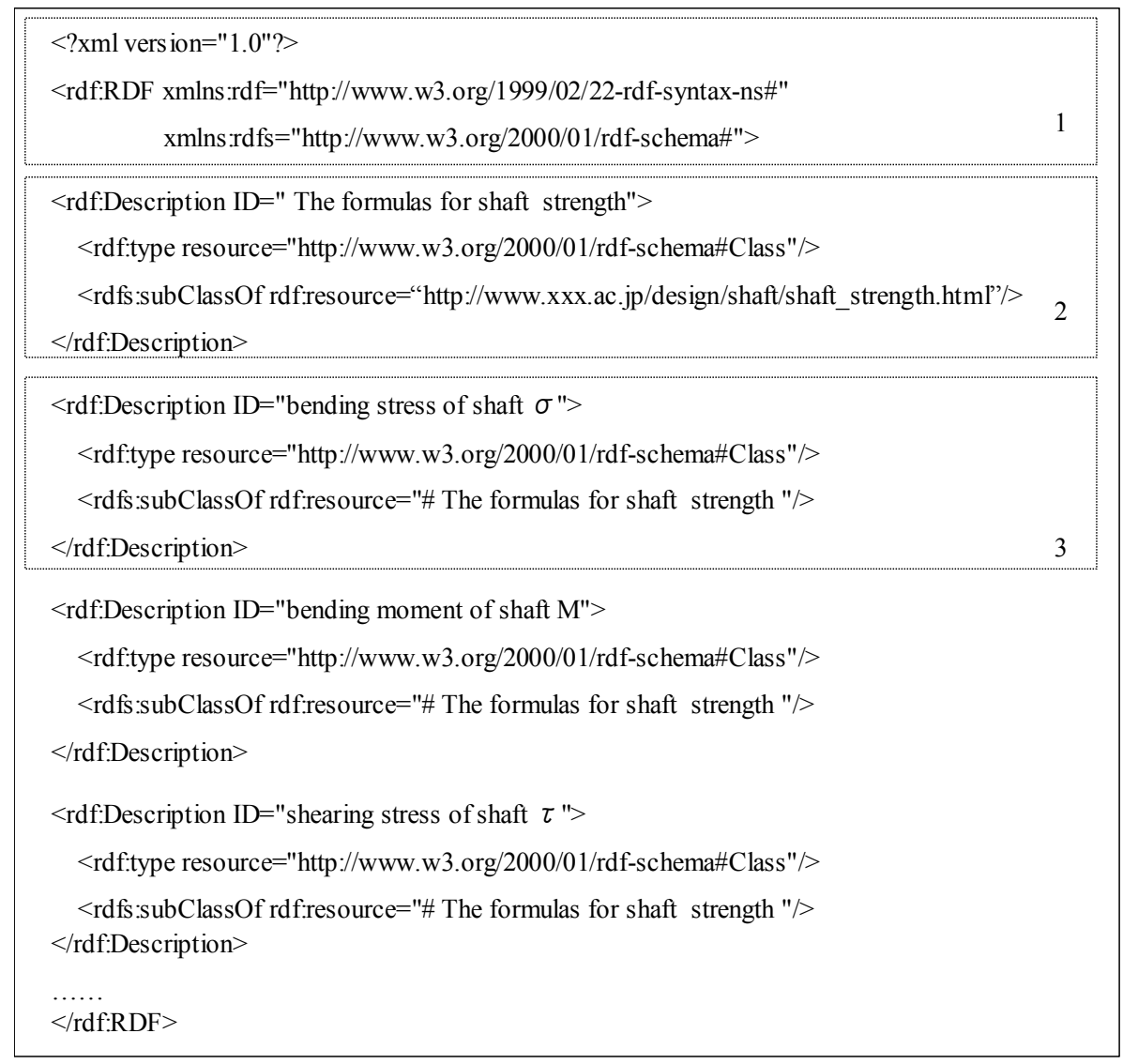

\section{Sharing of mechanical design formula data}

3.1 MathML for the expression of mechanical design formula data

MathML ${ }^{(11)}$ is an XML based markup language that is used to express mathematical formulas. It was developed by $\mathrm{W} 3 \mathrm{C}$ for the purposes of displaying mathematical formulas effectively on web pages, and enabling easy data sharing between applications and reuse of mathematical content. MathML can be divided into two types: presentation markup and 
content markup. The first type is mainly used for visual expression while the latter is used for data processing. Prior to the existence of MathML, mathematical formulas were expressed using pictures, text, and other formats that do not retain mathematical meaning.

Table 1 MathML presentation elements for mathematical formulas

\begin{tabular}{|c|l|}
\hline Element & \multicolumn{1}{|c|}{ Description } \\
\hline mrow & The mrow element is used to group together any number of sub-expressions. \\
\hline $\mathrm{mi}$ & $\begin{array}{l}\text { The mi element represents a symbolic name or arbitrary text that should be rendered as an } \\
\text { identifier. Identifiers can include variables, function names, and symbolic constants. }\end{array}$ \\
\hline $\mathrm{mo}$ & The mo element represents an operator or anything which should be rendered as an operator. \\
\hline $\mathrm{mn}$ & $\begin{array}{l}\text { The mn element represents a "numeric literal" or other data that should be rendered as a numeric } \\
\text { literal. }\end{array}$ \\
\hline musp & The msup element is used to attach a superscript to a base. \\
\hline mfrac & $\begin{array}{l}\text { The mfrac element is used for fractions. It can also be used to mark up fraction-like objects such } \\
\text { as binomial coefficients and Legendre symbols. }\end{array}$ \\
\hline
\end{tabular}

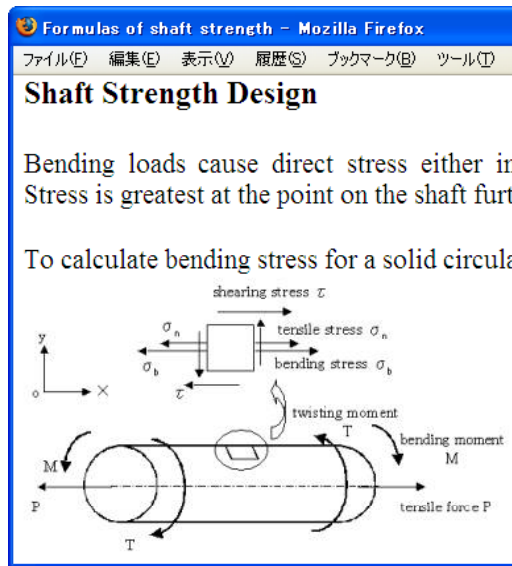

MathML document

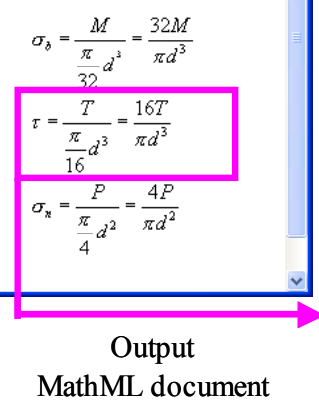

Figure 4 Display of a MathML document by Mozilla browser

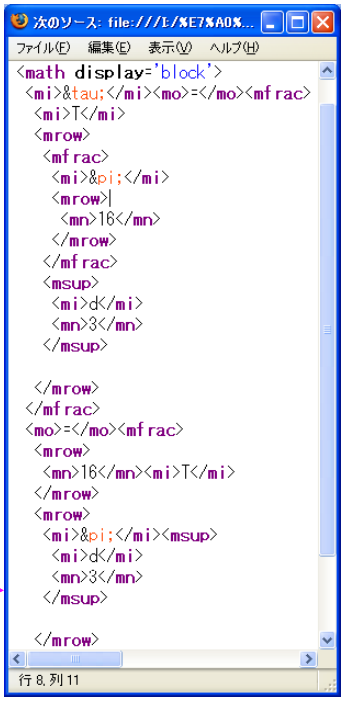

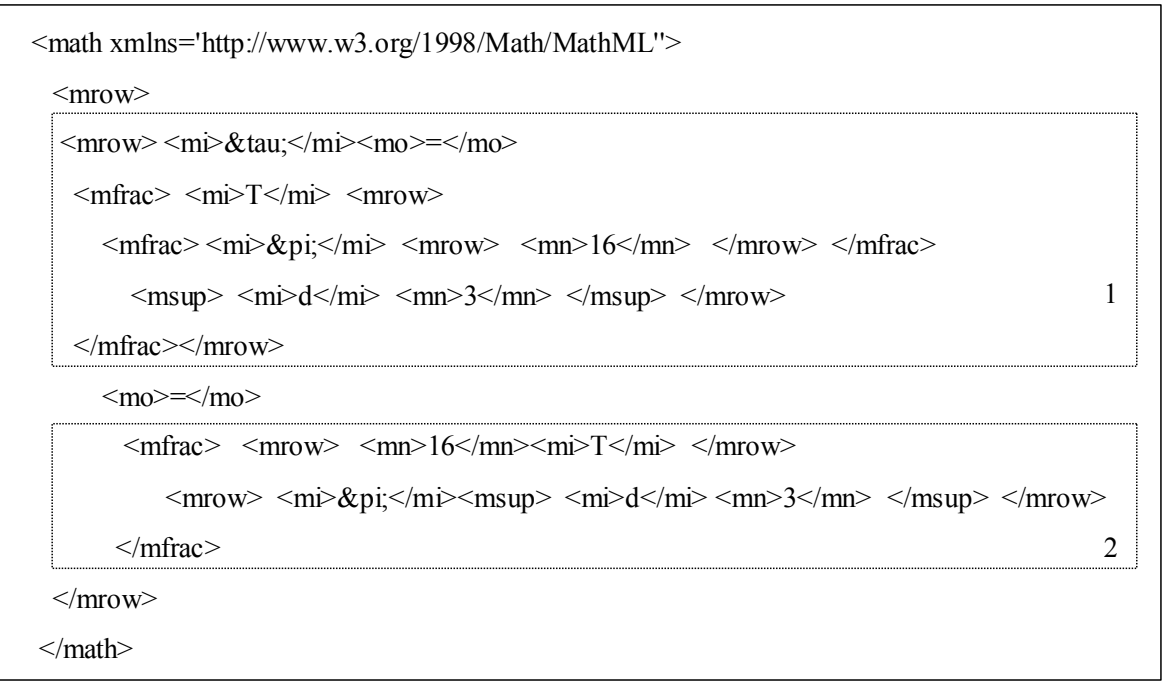


In this paper, MathML presentation markup is used for the example expressing "formulas for shaft strength". Table 1 shows the MathML presentation elements for mathematical formulas. In order to inspect web pages that contain MathML, browsers that can display MathML are necessary. Because only Mozilla and Amago are good for the direct presentation of MathML at present, experiments were conducted using a Mozilla browser in this study. Figure 4 shows the mechanical design formulas for shaft strength written in MathML and displayed on a Mozilla browser.

List 3 is an example MathML document. In this paper, "formulas for shaft strength" are converted into MathML using the MathType ${ }^{(17)}$ software.

MathML documents are explained below. In all MathML documents, the most super-ordinate concepts are math elements, and name spaces are used to clarify their meanings. Whole formulas are enclosed within math elements. These are the root elements for the entirety of MathML markup. The example of "formulas for shaft strength" is represented according to Table 1. $\sigma$ is described by "\&sigma;" and multiplication is described by "\&times;". Part (1) of the MathML document is stated as $\tau=\frac{T}{\frac{\pi}{16} d^{3}}$ and part (2) as $\frac{16 T}{\pi d^{3}}$.

3.2 Cooperation between the Semantic Web and MathML

At present, there have been some attempts to construct mathematical expression search engines on the Web. Kishimoto et al. ${ }^{(18)}$ presented an implementation method for similarity-based retrieval of mathematical formulas described in MathML which enables the acquisition of similar mathematical formulas by using MathML expressions as the queries. Murakata et al. ${ }^{(19)}$ realized a complex mathematical formula search handling both language and mathematical formulas. However, constructing search engines for the next generation web by describing mathematical expressions in MathML format has not yet been proposed.

In this paper, the mechanical design formulas are written in the MathML language, and the associated metadata are thus determined. In this case, the metadata are equivalent to the mechanical design formulas defined by MathML. The definition of the metadata for the formulas and the relationships among the metadata are defined using the RDF Schema language by attaching RDF documents and RDF Schema to the MathML code. Later, the data described by RDF Schema are saved on the server side to enable mechanical processing. Data sharing of mechanical design formulas is made possible by the cooperation between the Semantic Web and MathML.

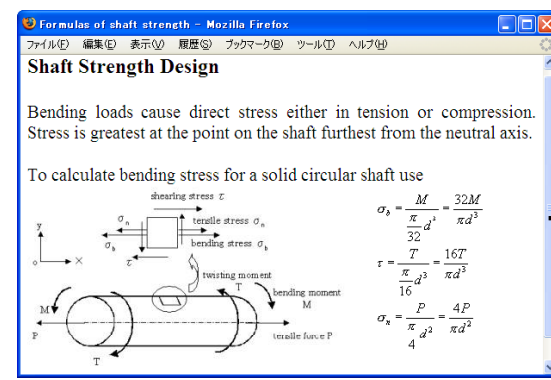

(a) Webpage

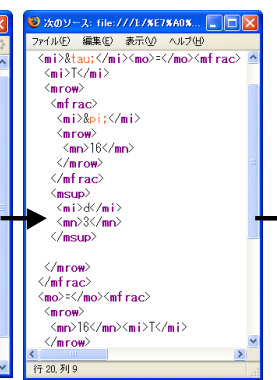

(b) Mechanical design formulas using MathM

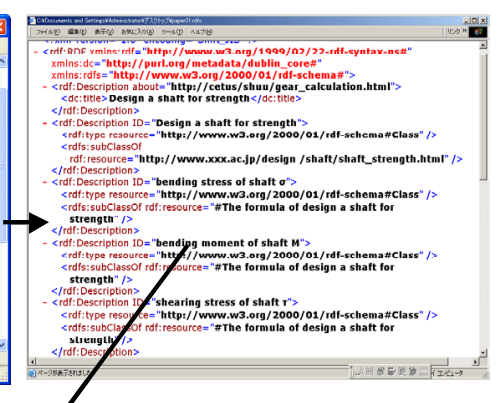

(c) RDFS document

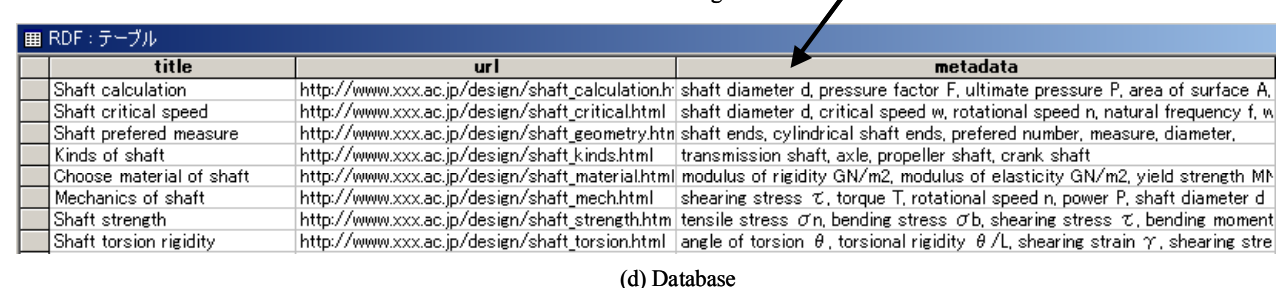
(d) Database

Figure 5 Construction of a database for mechanical design 
As shown in the example depicted in Figure 5, "formulas of shaft strength" are described in the MathML language on the Internet. The expressions defined using MathML are written in the RDF Schema language, and the related metadata are all well defined and saved in the server-side database. Keywords are input by the user into the search engine. Accurate search results can be obtained by having the server process the RDF Schema.

\section{Database on the Semantic Web}

At the present time, APIs for languages such as Java and Perl have been developed ${ }^{(20)}$ in order to deal with RDF, e.g., Jena and RQL ${ }^{(21)}$. We performed here an adequacy search in which metadata defined by RDF and RDFS documents were built into a simpler database.

Part (d) of Figure 5 represents an example database construction. The database on the Semantic Web is constructed using RDF elements. This database includes the data in the table, the rows of which are records, and the columns of which are fields. The relationship between the elements of RDF documents and the table data are as follows.

(1) The record with field name "title" is an RDF attribute.

(2) The record with field name "url" is an RDF resource.

(3) The record with field name "metadata" is the RDF description.

A search engine is needed when constructing semantic applications. As shown in Figure 6, we made an accurate search for a design formula by extracting information from the database using Active Server Pages (ASP) ${ }^{(22)}$ technology.

The index.asp file offers an input box for keyword-based searching. When the keywords are input and the search button is pressed, the result.asp file displays the search results based on the search conditions specified in index.asp. After keywords have been input, and the user has pushed the search button, the result.asp file expresses the search results based on the search conditions specified in index.asp. The disp.asp file is used to access the database and the result.asp file.

For example, when the keywords "design of shaft" and "calculation of shaft strength" are input, pages related solely to the size calculation of the shaft, or related solely to shaft design are not retrieved, instead only those related to both are retrieved.

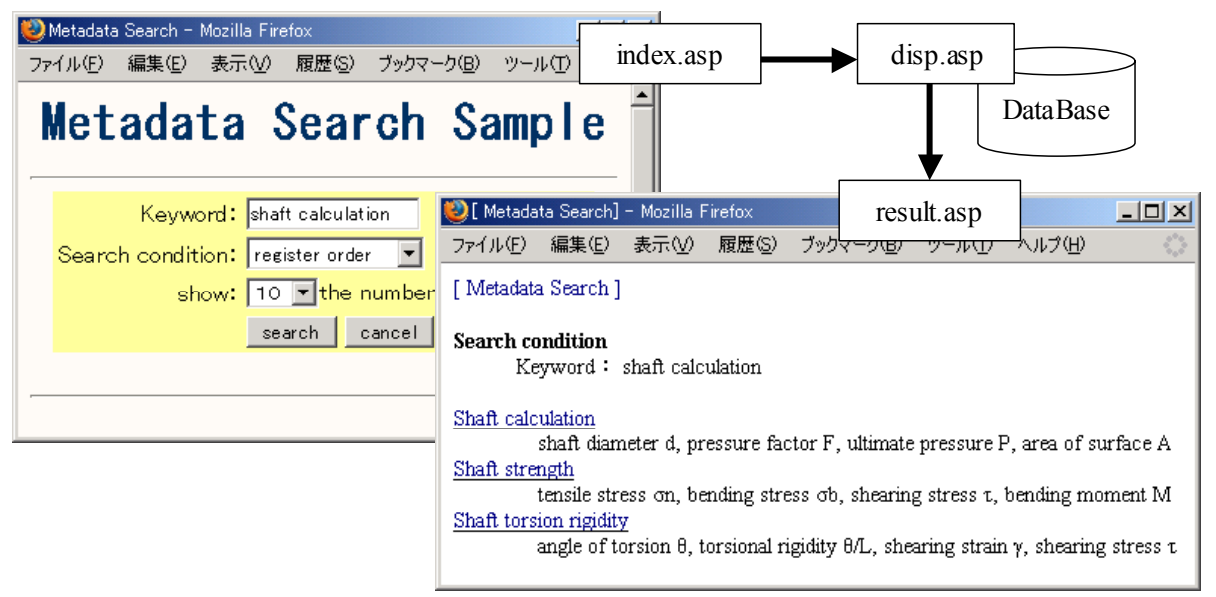

Figure 6 Search engine

\section{Application example}

5.1 Calculating mechanical design formulas using the mathematical formula processing system

Mathematica is a mathematical formula processing system that includes formula 
processing, numerical computation, and graphical display functions ${ }^{(23)}$. Content written in Mathematica format can be directly converted into MathML format. Furthermore, content is interchangeable simply by using copy and paste. Web designers can therefore write and evaluate design formulas on Mathematica, and subsequently convert this content into MathML format. The converted content is later copied into XML or XHTML pages to enable the mathematical expressions to be displayed on the web.

Calculations were performed for this paper using a mathematical formula processing system. Figure 7 shows a web page containing related technical documents, in which the design formulas are linked to the Mathematica software. The design formulas are calculated automatically by activating Mathematica and keying in the design formulas and related parameter values. The results calculated can be carried forward to the next calculation when necessary. Finally, the results can be saved as MathML documents as required. The shaft stress calculation results are shown using Mathematica software. As described above, the calculation is rendered straightforward, and the efficiency of the design task is improved. The sharing of mechanical design formula data is thus achieved through cooperation between the Semantic Web, MathML and Mathematica.

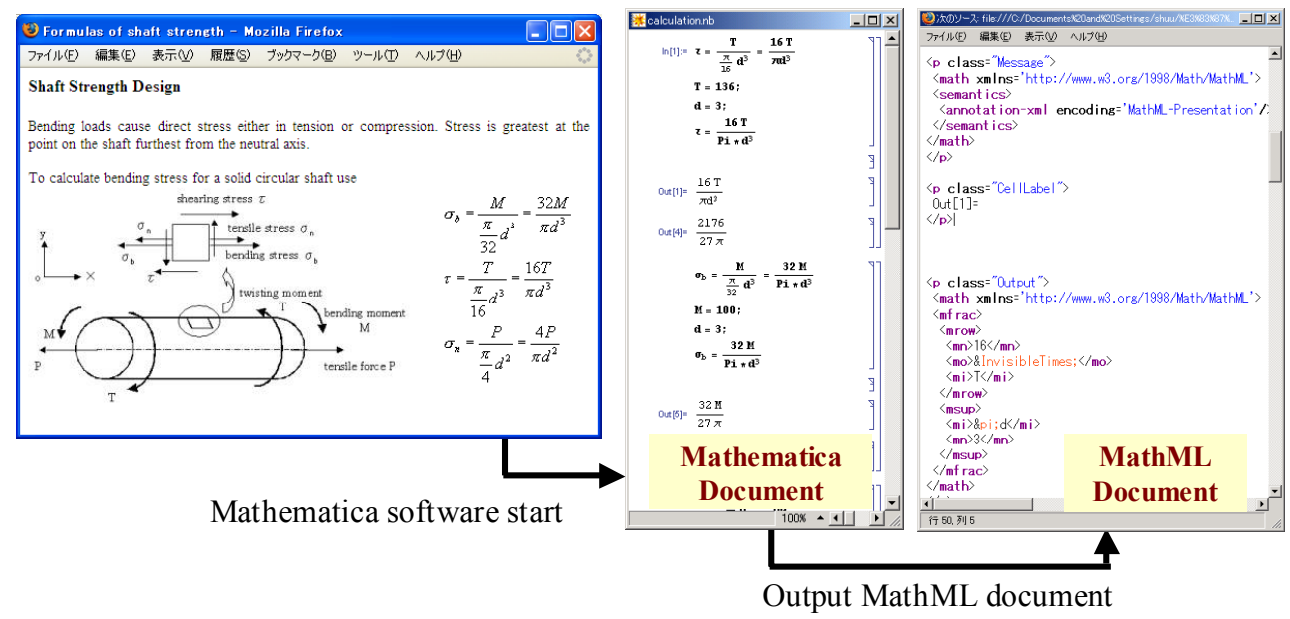

Figure 7 Shaft calculation in Mathematica

on the server side

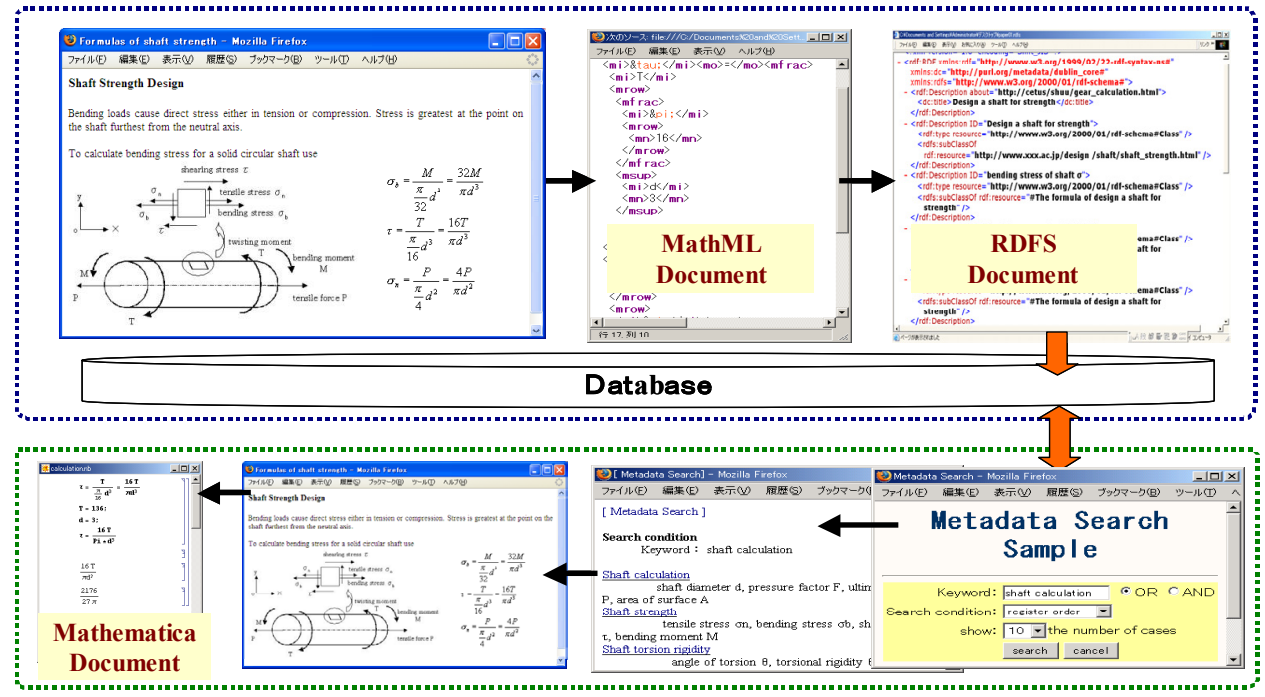

on the user side

Figure 8 Outline of a data sharing support system for mechanical design formulas 


\subsection{System composition}

Semantic Web technology plays an important role in the processing, sharing, and reuse of knowledge on the web. However, a generally accepted methodology for system development remains to be established. It is therefore necessary to establish a construction methodology for the Semantic Web and build a corresponding system development kit (SDK). This paper is the first attempt to build of a search engine for the next generation web by expressing mathematical formulas using MathML documents. Hierarchical concepts of knowledge consisting of mechanical design documents and formulas were analyzed in this paper, and described in the RDFS language of the Semantic Web. A database for the Semantic Web can be developed based on this RDFS data.

We have also proposed a construction methodology, whereby mechanical designs are written in MathML to enable data sharing on the Semantic Web. Figure 8 shows the GUI of our proposed system.

By developing the Semantic Web database, web searching can be rendered very effective with the combined usage of RDB/ASP and the Web. Furthermore, as the design formulas are described in the MathML language, calculation can be made easy using Mathematica software. It has thus been made possible to share design formula data using the Semantic Web.

\section{Conclusion}

In this paper, we have proposed the sharing of mechanical design formula data using the Semantic Web. This enabled design formula data to be shared globally, and rendered searching for accurate information more effective. Furthermore, it has become clear that the broad abundance of mechanical design information may accordingly be placed into a database.

\section{References}

(1) Berners-Lee, T., Hendler, J. and Lassila, O.: The Semantic Web, Scientific American, May (2001), 34.

(2) Decker, S., Melnik, S., Harmelen, F.V., Fensel, D., Klein, M., Broekstra, J., Erdmann, M. and Horrocks, I.: The Semantic Web: The Roles of XML and RDF, IEEE Internet Computing, 4, 5 (2000), 63.

(3) Knowledge Engineering Methods and Languages ftp://swi.psy.uva.nl $/ \mathrm{pub} / \mathrm{keml} / \mathrm{keml} . \mathrm{html}$

(4) Problem-Solving Methods Mailing List: http://www.swi.psy.uva.nl/mailing-lists/kaw-psm/home.html

(5) Ontology: http://mnemosyne.itc.it:1024/ontology.html

(6) HPKB: High Performance Knowledge Bases http://www.teknowledge.com/HPKB/

(7) IBROW3: An Intelligent Brokering Service for Knowledge-Component Reuse on the World-Wide Web http://www.swi.psy.uva.nl/projects/IBROW3/home.html

(8) K.R. Foster, "Math on the Internet," IEEE Spectr., vol.36, no.4, April 1999.

(9) Watanuki, K., Zhou, J.: Structuring and Sharing of Mechanical Design Knowledge Using Ontology Technology, In Proc. of JSME 10th Kanto Branch Annual Meeting, No.040-1, pp.491-492, (2003) (in Japanese).

(10) Zhou J., Watanuki K.: Structuring and Sharing of Mechanical Design Knowledge Using Semantic Web Technology, Proceedings of the First Asia International Symposium on Mechatronics (AISM 2004), (2004), pp.269-274.

(11) Sandhu, P.: The MathML Handbook, Charles River Media Pub. (2002), 518.

(12) Manola F. and Miller E. (eds.): RDF Primer, W3C Recommendation, (2004).

(13) RDF Vocabulary Description Language 1.0: RDF Schema, http://www.w3.org/TR/rdf -schema/ 
(14) Wolfram, S.: MATHEMATICA5, Wolfram Media (2003), 1424.

(15) Birbeck, M.: Professional XML Meta Data, Wrox Press (2001).

(16) Dublin Core Metadata Initiative: Dublin Core Metadata Element Set, Version 1.1: Reference Description. Retrieved June 15, (2003), from http://dublincore.org/documents/dces

(17) MathType: http://www.chartwellyorke.com/mathtype.html

(18) S.Kishimoto, T.Nakanishi, T.Sakurai, T.Kitagawa: An Implementation Method of Similarity-Based Retrieval for Formulas using MathML, Date Engineering Workshop (DEWS2003), no.6-P-07. (in Japanese).

(19) M.Murakata, S.Kishimoto, T.Otsuka, T.Nakanishi, T.Sakurai, T.Kitagawa: Realization of the input support GUI "MathGUIde" for a complex mathematical formulas search, Date Engineering Workshop (DEWS2006), no.6B-i7. (in Japanese).

(20) Powers, S.: Practical RDF, O’Reilly Pub. (2003), 171.

(21) Karvounarakis, S., Magkanaraki, A., Alexaki, S., Christophides, V., Plexousakis, D., Scholl, M. and Tolle, K.: RQL: A Functional Query Language for RDF, Functional Approaches to Computing With Data, Gray, P.M.D., Kerschberg, L., King, P.J.H. and Poulovassills, A. (eds.), LNCS Series, Springer-Verlag (2003), 1.

(22) Active Server Pages: http://en.wikipedia.org/wiki/Active_Server_Pages

(23) Totigi, M., Kimura, K., Sakurai, T.: Mathematical principle document use support by web application, The 31st Numerical Analysis Symposium, NSA2002, 31 (2002), 125 (in Japanese). 